\title{
Stability-indicating HPLC method for isoflavones aglycones analysis from Trifolium pratense L. extract
}

\author{
Simony Martiny ${ }^{{ }^{a}}$, Mairique Waszczuk ${ }^{\mathrm{a}}$, Samuel Kaiser ${ }^{\mathrm{a}}$, Marina Cardoso Nemitz ${ }^{\mathrm{b}}$, Valquiria Linck Bassani ${ }^{\mathrm{a}}$ \\ ${ }^{a}$ Programa de Pós-Graduação em Ciências Farmacêuticas, Faculdade de Farmácia, Universidade Federal do Rio Grande do Sul \\ (UFRGS), Porto Alegre, RS, Brazil; ${ }^{b}$ Faculdade de Farmácia, Universidade Federal do Rio de Janeiro (UFRJ), Macaé, RJ, \\ Brazil.
}

*Corresponding author: simony.martiny@gmail.com

\begin{abstract}
The purpose of this study was to develop and validate a fast HPLC stability-indicating method for simultaneously quantifying the four main isoflavones in Trifolium pratense. Validation procedures followed the ICH and FDA requirements for complex matrices. The stability-indicating tests were performed by exposing the isoflavones to conditions of forced degradation and further analysis for verifying the formation of degradation products and their possible interferences in the HPLC analysis. The major isoflavones of Trifolium pratense proved to be stable against acid and oxidative media, thermodegradation, and photodegradation. However, they proved to be unstable in alkaline media, even for short periods of exposure like $2 \mathrm{~h}$. In this condition, in addition to the peaks corresponding to isoflavones, the HPLC analysis showed the presence of three additional peaks which were eluted at different retention times to the reference substances, without interfering in the quantification of the four analytes of interest, formononetin, biochanin A, daidzein, and genistein. The method was validated following ICH and FDA guidelines showing to be specific, linear, precise, accurate, and robust. This first report concerning a stability-indicating method revealed that the proposed HPLC method reliably quantify the isoflavones and separate them from the degradation products in a short time of analysis.
\end{abstract}

Keywords: Trifolium pratense, isoflavone aglycones, HPLC, stability-indicating method, validation DOI: https://doi.org/10.22456/2527-2616.102027

\section{Introduction}

Trifolium pratense L. (red clover) is a species that contains isoflavones, and its extracts are widely used as phytoestrogens, mainly due to their beneficial effects on climacteric symptoms. In order to expand the range of therapeutic applications, other effects that arouse interest have been investigated, such as antioxidants, anti-tumor, antibacterial, anti-inflammatory, and antifungal. [1-5].

Some methods have been reported to quantify the major isoflavones in Trifolium pratense products formononetin (FORMO), biochanin A (BIO), daidzein (DAID) and genistein (GEN) [4,6-11]. Most of these methods use highperformance liquid chromatography (HPLC) to perform the isoflavones quantification taking a long time of analysis (most of them more than 30 min considering the carry-over effect). Moreover, none of these studies consider the possibility of interference of degradation products on the quantification of the analytes.

In this context, this study aimed to develop and validate a fast HPLC stability-indicating method for simultaneously quantifying the four main isoflavones in Trifolium pratense, FORMO, BIO, DAID, and GEN. The validation was performed following the ICH and FDA requirements for complex matrices. The stability-indicating tests were carried out by exposing the isoflavones to conditions of forced degradation (stress-induced by alkaline, acid, oxidative, and light exposure conditions) and further HPLC analysis for verifying if the degradation products interfere in the assay of the analytes. The degradation products had their fragmentation patterns determined using mass spectrometry. To the best of our knowledge, these findings are being reported for the first time being original and relevant to their application in both food and pharmaceutical fields.

\section{Material and Methods}

\section{Chemical and materials}

The isoflavones used as reference substances (standards), biochanin A, daidzein and genistein $(\geq 98 \%, \geq 95 \%$ and $\geq 98 \%$ purity, respectively) were purchased from Cayman Chemical Company (Ann Arbor, MI, EUA); and formononetin ( $\geq 98 \%$ purity) was purchased from Acros Organics (Belgium). Acetonitrile (HPLC grade, Tedia, Fairfield, USA), trifluoracetic acid (Vetec, Brazil) and water purified by a Milli-Q water system (Millipore, Bedford, MA, USA) were used in the HPLC analysis.

\section{Apparatus and analytical conditions}

\section{HPLC Analysis}

The development and validation of the HPLC method were performed on a Shimadzu HPLC-20A (Kyoto, Japan), consisting of an HPLC-20AT pump, a SIL-20AT autosampler, and a variable wavelength (UV/vis) SPD-M20A detector. The data acquisition and treatment was performed using a Shimadzu HPLC Solution GPC software (Kyoto, Japan). A CLC-ODS (4.6 mm X $25 \mathrm{~cm}$ i.d; $5 \mu \mathrm{m}$ ) shim-pack column (Shimadzu, Kyoto, Japan) coupled to a C18 precolumn (20 X 3.9 mm i.d; 10mm) (Waters, Milford, MA, USA) were employed.

The mobile phase of the proposed chromatographic method consisted of a phase A composed of water acidified with $0.1 \%$ of trifluoroacetic acid (TFA) and a phase B composed of acetonitrile. The gradient elution was 30\% B (0-2 min), 30- 
$55 \% \mathrm{~B}(2-13 \mathrm{~min})$, and $55-30 \% \mathrm{~B}$ (13-19 $\mathrm{min})$, while the flow rate was a gradient of $1.0 \mathrm{~mL} / \mathrm{min}(0-11.7 \mathrm{~min}), 1.0-0.4$ $\mathrm{mL} / \mathrm{min}$ (11.7-12.2 $\mathrm{min}), 0.4 \mathrm{~mL} / \mathrm{min}$ (12.2-13.8 $\mathrm{min}), 0.4$ $1.0 \mathrm{~mL} / \mathrm{min}$ (13.8-14.2 $\mathrm{min})$, and $1.0 \mathrm{~mL} / \mathrm{min}$ (14.2-19 $\mathrm{min})$. The column was re-equilibrated with $30 \%$ phase B for two minutes before the next analysis was initiated. The injection volume was $10 \mu \mathrm{L}$, the analysis temperature was $40^{\circ} \mathrm{C}$, and the UV detector was set at wavelength $260 \mathrm{~nm}$.

\section{Mass Spectrometry (MS)}

Isoflavone aglycones stability-indicating studies were performed using an I-Class ACQUITY ultra-performance liquid chromatography (UPLCTM, Waters, USA) system (Waters, USA) coupled to a quadrupole time-of-flight analyzer (QTOF) (SYNAPT G2 Si, Waters, USA). The UPLC analysis parameters were based on the HPLC analytical conditions, with modifications. An Acquity UPLC HSS T3 column $(2.1 \times 10 \mathrm{~mm}$ i.d., $1.8 \mu \mathrm{m})$ (Waters, USA) was used. The mobile phase was a formic acid solution $0.1 \%$ $(\mathrm{v} / \mathrm{v})(\mathrm{A})$, and acetonitrile with formic acid $0.1 \%(\mathrm{v} / \mathrm{v})(\mathrm{B})$ following a gradient elution program: $0-1 \mathrm{~min}, 30 \% \mathrm{~B} ; 1-8$ min, from $30 \%$ to $60 \% \mathrm{~B} ; 8-9.5 \mathrm{~min}, 60 \% \mathrm{~B}$; 9.5-9.6 min, from $60 \% \mathrm{~B}$ to $30 \% \mathrm{~B} ; 12 \mathrm{~min}$, stop time. The flow rate was $0.4 \mathrm{~mL} / \mathrm{min}$, the injection volume was $0.6 \mu \mathrm{L}$ and the column temperature was $40^{\circ} \mathrm{C}$. A leucine-encephalin solution (1 $\mu \mathrm{g} / \mathrm{mL}$; acetonitrile:water $(50: 50, \mathrm{v} / \mathrm{v})$ with formic acid $0.1 \%$ $(\mathrm{v} / \mathrm{v})$ ) was employed as a lock mass solution $(554.2615 \mathrm{~m} / \mathrm{z}$ in ESI- and $556.2771 \mathrm{~m} / \mathrm{z}$ in ESI+). The data were processed with Mass Lynx 4.1 and Mass fragment 3.0 software (Waters, USA). The analysis was performed in ESI- and ESI+ MSE centroid mode, in TOF mode (Resolving power 20,000). The source conditions were: $2 \mathrm{kV}$ of capillarity; $120^{\circ} \mathrm{C}$ and $550^{\circ} \mathrm{C}$ source and desolvation temperatures, respectively; $30 \mathrm{~V}$ and $80 \mathrm{~V}$ for the sampling cone and source offset, respectively; $50 \mathrm{~L} / \mathrm{h}$ and $900 \mathrm{~L} / \mathrm{h}$ of cone and desolvation gases flow, respectively; and 6.5 bar of spray gas flow. Nitrogen was used as a desolvation gas, cone gas, and nebulizer gas. The argon was employed as collision gas at transfer $(40 \mathrm{eV})$ collision cells. A $50-1000 \mathrm{~m} / \mathrm{z}$ mass range was monitored with a scan time of $0.2 \mathrm{~s}$ and an interscan delay of $0.015 \mathrm{~s}$.

\section{Solutions}

\section{Stock and reference solutions}

Stock isoflavone solutions $(80 \mu \mathrm{g} / \mathrm{mL})$ FOR, BIO, DAID, and GEN were prepared in acetonitrile by weighing approximately $8 \mathrm{mg}$ of each component into $100 \mathrm{~mL}$ calibrated volumetric flasks. Reference solutions were prepared by dilutions of the stock solution with an aqueous solution of $50 \%(\mathrm{v} / \mathrm{v})$ acetonitrile.

\section{Trifolium pratense extract (matrix)}

Trifolium pratense extract was previously obtained by maceration in $40 \%$ ethanol at $25^{\circ} \mathrm{C}$ for 7 days $(1: 15 \mathrm{w} / \mathrm{v})$. A suitable aliquot $(1: 5 \mathrm{v} / \mathrm{v})$ was diluted in an aqueous solution of $50 \%(\mathrm{v} / \mathrm{v})$ acetonitrile, filtered through a $0.45 \mu \mathrm{m}$ membrane and analyzed.

\section{Validation of the analytical method}

The developed method was validated according to the official validation guidelines $[12,13]$ in a range of $0.5-20 \mu \mathrm{g} / \mathrm{mL}$ for FORMO, BIO, DAID, and GEN. The results were analyzed by Student's t-test and analysis of variance (ANOVA) using a level of significance of $\alpha=0.05$. The interference of degradation products formed in the stability-indicating tests on the Trifolium pratense major isoflavones assay was also evaluated in the validation.

\section{Assessment of the matrix effect}

The matrix effect evaluation was performed as described by Watanabe et al. [14] and Yatsu et al. [15] by comparing the slopes of calibration curves of the isoflavone aglycone standards (FOR, BIO, DAID, and GEN) in the solvent and the matrix.

Three analytical curves were obtained on three consecutive days by plotting the peak areas versus the concentration of isoflavone aglycone standards $(0.5,1.0,4.0,8.0,12.0,16.0$, and $20.0 \mu \mathrm{g} / \mathrm{mL})$ in acetonitrile $50 \%(\mathrm{v} / \mathrm{v})$ and the matrix solution. Each concentration level was analyzed in five replicates.

The matrix effect was calculated according to the following equation: $\quad M E \%=100 \times[1-(\mathrm{Sm} / \mathrm{Ss})]$, where $\mathrm{Sm}$ corresponds to the slope of the standard curves of the isoflavone standards in the mobile phase and $S s$ is the slopes of the standard curves of the isoflavone standards in the matrix.

\section{Specificity and stability-indicating tests}

The specificity of the HPLC method was evaluated by injecting samples containing only the matrix (Trifolium pratense extract) and samples containing the matrix added of isoflavone aglycones standard quantities of FORMO, BIO, DAID, and GEN in concentrations of $4 \mu \mathrm{g} / \mathrm{mL}$ each one. The aglycone isoflavones present in the matrix were identified based on their UV spectra, between 200 and $400 \mathrm{~nm}$, and their retention times compared to the reference substances.

For testing the specificity against cyclodextrins, an amount of approximately $1 \mathrm{mg}$ of hydroxypropyl-beta-cyclodextrin (HP $\beta C D)$ and beta-cyclodextrin ( $\beta C D)$ was dissolved with an aqueous solution of acetonitrile $(50 \% \mathrm{v} / \mathrm{v})$ in $25 \mathrm{ml}$ volumetric flasks. These samples were placed in an ultrasound bath for 30 minutes, filtered in $0.45 \mu \mathrm{m}$ membrane and injected in HPLC equipment.

The specificity of the HPLC method against the degradation of products from stability-indicating tests was also evaluated. The stock solutions of standards were also submitted to the forced degradation conditions shown in Table 1.

All samples were compared to a control solution of the analytes (FORMO, BIO, DAID, and GEN) at a concentration of $16 \mu \mathrm{g} / \mathrm{mL}$ (diluted in an aqueous solution of acetonitrile $50 \% \mathrm{v} / \mathrm{v})$, protected from light. The analysis was carried out in triplicate, and the purity of the peak was determined using a PDA detector. Mass spectrometry analysis was performed for the degradation products after forced degradation.

Table 1. Conditions of forced degradation tests for the isoflavones of Trifolium pratense. 


\begin{tabular}{lccc}
\hline Degradation medium & Reagents & $\begin{array}{c}\text { Temperature } \\
\left({ }^{\circ} \mathbf{C}\right)\end{array}$ & $\begin{array}{c}\text { Time points } \\
(\mathbf{h r s})\end{array}$ \\
\hline Acid degradation & $1 \mathrm{M} \mathrm{HCl}$ & 25 & $2 ; 24$ \\
Alkaline degradation & $1 \mathrm{M} \mathrm{NaOH}$ & 25 & $2 ; 24$ \\
\cline { 2 - 4 } Oxidation & $\mathrm{H}_{2} \mathrm{O}_{2} 1.5 \%$ & 25 & $2 ; 24$ \\
& $\mathrm{H}_{2} \mathrm{O}_{2} 15 \%$ & 25 & $2 ; 24$ \\
\cline { 2 - 4 } Thermal & - & 80 & $12 ; 24$ \\
Photostability & $\mathrm{UVC}^{\mathrm{a}}$ & 25 & $24 ; 48$ \\
\hline
\end{tabular}

${ }^{a}$ UVC Light Express LE, 245 nm, 30W

\section{Linearity, precision and accuracy}

The linearity of the method was evaluated by regression analysis using the least square method. Three calibration curves were obtained by performing dilutions from the reference isoflavone stock solution on three consecutive days. The plot of the peak area versus the reference aglycone isoflavone concentration, diluted in $50 \%(\mathrm{v} / \mathrm{v})$ acetonitrile, was performed. The linearity of the method was given in the following concentrations: $0.5,1.0,4.0,8.0,12.0,16.0$ and $20.0 \mu \mathrm{g} / \mathrm{mL}$ for the reference aglycones and $0.5,1.0,2.0,4.0$, $6.0,8.0$, and $10.0 \mu \mathrm{g} / \mathrm{mL}$ in matrices. For each level of concentration, samples were taken in six replicates. The limits of detection (LOD) and quantification (LOQ) of the method were calculated based on the standard deviation of the intercept and slope of the reference curves.

The intra-day precision, which evaluates the repeatability of the results, was determined in three concentration levels: low, medium, and high $(1.0,4.0$, and $8.0 \mu \mathrm{g} / \mathrm{mL}$, respectively) in the presence of the matrix. Six analyses were performed for each concentration within the same day and under the same experimental conditions. The intermediate precision (interday precision) was obtained by calculating the same concentration levels on three different days. The standard deviation and the relative standard deviation (RSD) were calculated for each of these levels. To assess the accuracy of the method, isoflavone aglycone standard was added at three different amounts/levels (1.0, 4.0 and $8.0 \mu \mathrm{g} / \mathrm{mL})$ to the matrix. For each concentration level, six replicates were performed and quantified. Accuracy was determined mathematically through the equation:

$$
R E \%=\frac{\text { mean calculated concentration }- \text { theoretical value }}{(\text { theoretical value }) \times 100}
$$

\section{System suitability}

The suitability test of the analytical system was performed to determine whether the method was suitable for the proposed analysis. The parameters evaluated were the retention time, peak area, plates, tailing factor, and resolution between the FORMO, BIO, DAID, and GEN peaks. Each parameter was analyzed in six replicates.

\section{Robustness}

Robustness was assessed according to the Plackett-Burmann design method, as described by Vander Heyden et al [16]. The factors analyzed for the matrix, which were modified to low $(-1)$ and high (+1) levels, were: furnace temperature of the column $\left(39 ; 41^{\circ} \mathrm{C}\right)$, acetonitrile concentration in the mobile phase $(29 ; 31 \%)$, initial flow rate $(0.9 ; 1.1 \mathrm{~mL} / \mathrm{min})$ and TFA concentration $(0.08 ; 0.12 \%)$. The variation of these four factors were combined in eight experiments and the responses evaluated were the percentages of aglycone isoflavones (FORMO, BIO, DAID, and GEN) in the matrix compared to the standard solutions. The effect of each parameter was calculated by summing the responses of the low and high levels, allowing the statistical interpretation ( $\left.t_{\text {calculated }}>t_{\text {critical }}\right)$ of the similarity or difference of the results. There was no need for further testing since all isoflavone standard aglycones and matrixes were maintained under the same experimental conditions. A plot of semi-normal probability was also plotted to estimate the experimental error and significant effects from the effects in combination with the dummy factors.

\section{Stability of the aglycones in the matrix}

The stability of the isoflavones in the matrix added with known amounts of isoflavone standards was determined after $48 \mathrm{~h}$ of storage at room temperature. The stability of these solutions was assessed by analyzing any change in the chromatographic profile compared to freshly prepared solutions.

\section{Method application}

The determination of FORMO, BIO, DAID, and GEN in a Trifolium pratense extract (TPE) was performed as described in the section matrix solution. Brief, an isoflavone-enriched fraction (IEF) was obtained by evaporation of a Trifolium pratense macerate, resulting in a dry fraction. This dry fraction was dissolved in a mixture of acetonitrile:water (1:1) to obtain $0.01 \% \mathrm{w} / \mathrm{v}$ solution. This solution was filtered through $0.45 \mu \mathrm{m}$ membrane (PTFE) and analyzed by HPLC. The determination of the aglycone isoflavones was further evaluated in a complex of the Trifolium pratense extract with $\beta$-cyclodextrin (TPE: $\beta C D)$. Spray drying method was employed for complexation, adding $\beta C D(40 \% \mathrm{w} / \mathrm{w})$ to the extract of Trifolium pratense (matrix). Approximately $1 \mathrm{mg}$ of this spray-dried powder was dissolved in a mixture of acetonitrile:water $(1: 1)$ to obtain a $0.01 \% \mathrm{w} / \mathrm{v}$ solution. The solution was filtered through a $0.45 \mu \mathrm{m}$ membrane (PTFE) and analyzed by HPLC.

\section{Results and discussion}

Many efforts for quantifying the isoflavones in Trifolium pratense raw material or derivatives products have been made in the last years generating several reports on HPLC analytical methods $[7,8,10,11,17]$. Despite that, some limitations remain. One of these is a long time of the analysis (> $30 \mathrm{~min}$ ) and the consequent high solvent consuming $[7,8,10,11,17]$. Another limitation is the occurrence of the matrix effect, which requires prior purification steps of the sample. However, what is especially striking is that no method alludes to the possibility of interference by degradation products in the quantification of the analytes, FORMO, BIO, DAID, and GEN. 
In this work, a new HPLC chromatographic method is proposed, which is faster than the methods previously reported, to determine the matrix effect and to identify and separate the Trifolium pratense aglycone isoflavones from the degradation products that are formed by degradation in the forced stability tests.

\section{HPLC method development and advantages}

The previous HPLC analytical methods are focused on separating the major isoflavones to quantifying them in different Trifolium pratense extracts [6-8,10,11,17]. However, these methods still require better performance such as lower elution time and the selectivity against the presence of products from forced degradation. Thus, in the present work, the method reported by Ramos et al. [7] was applied in order to separate the peak corresponding to the product formed in the alkaline degradation $(\mathrm{NaOH} 1 \mathrm{M}, 2 \mathrm{~h})$ from that corresponding to BIO. However, the peak corresponding to the degradation product showed similar retention time as FORMO and the HPLC-PDA analysis demonstrated that their UV spectra (PDA) were different (Fig. 1). This results demonstrated that the alkaline degradation of BIO produces a degradation product that interferes with the FORMO quantification, revealing the need to change the conditions of the HPLC analysis for separating them.

Thus, the stability-indicating method performed in the present work revealed the need to separate FORMO from the degradation product named DB for the reliable quantitation of FORMO; several chromatographic conditions were then tested. The best separation system for the analytes was achieved with a gradient method using acetonitrile (30\% v/v) and acidified water $(0.1 \% \mathrm{v} / \mathrm{v}$ TFA $)$, which improved the symmetry of the peaks. To improve the resolution among the peaks, it was necessary to use a flow gradient at the retention time where their separation was critical (11.7-14.2 min). The UV detection was set at $260 \mathrm{~nm}$ since all the aglycones isoflavones of interest have good absorption at this wavelength [18].

Figure 2 shows a representative chromatogram of the Trifolium pratense extract added with the alkaline degradation product of $\mathrm{BIO}$. Adequate separation of the peaks of all the analytes of interest (FORMO, BIO, DAID, and GEN) can be observed, as well the presence of the other peaks from the plant material and in relation to the BIO degradation peak (Table 2).

The total time of analysis of the proposed method was determined as being the eluate time plus the time necessary for cleaning the column before the next run. The method show 21 min of analysis time, which is shorter than the time analysis of more than $40 \mathrm{~min}$. reported by most of the methods (8, 10, 11 and 17). The carry-over effect was evaluated by analyzing white samples after the injection of samples with high concentrations of the analytes, and any peak in the chromatogram was observed. The method here proposed was characterized by absence of carry-over effect becomes the method here reported advantageous on the method reported by Ramos et al. [7], which present a run time of 18 minutes, but requires additional 18 minutes for cleaning the column and rebalance the system at each analysis end.

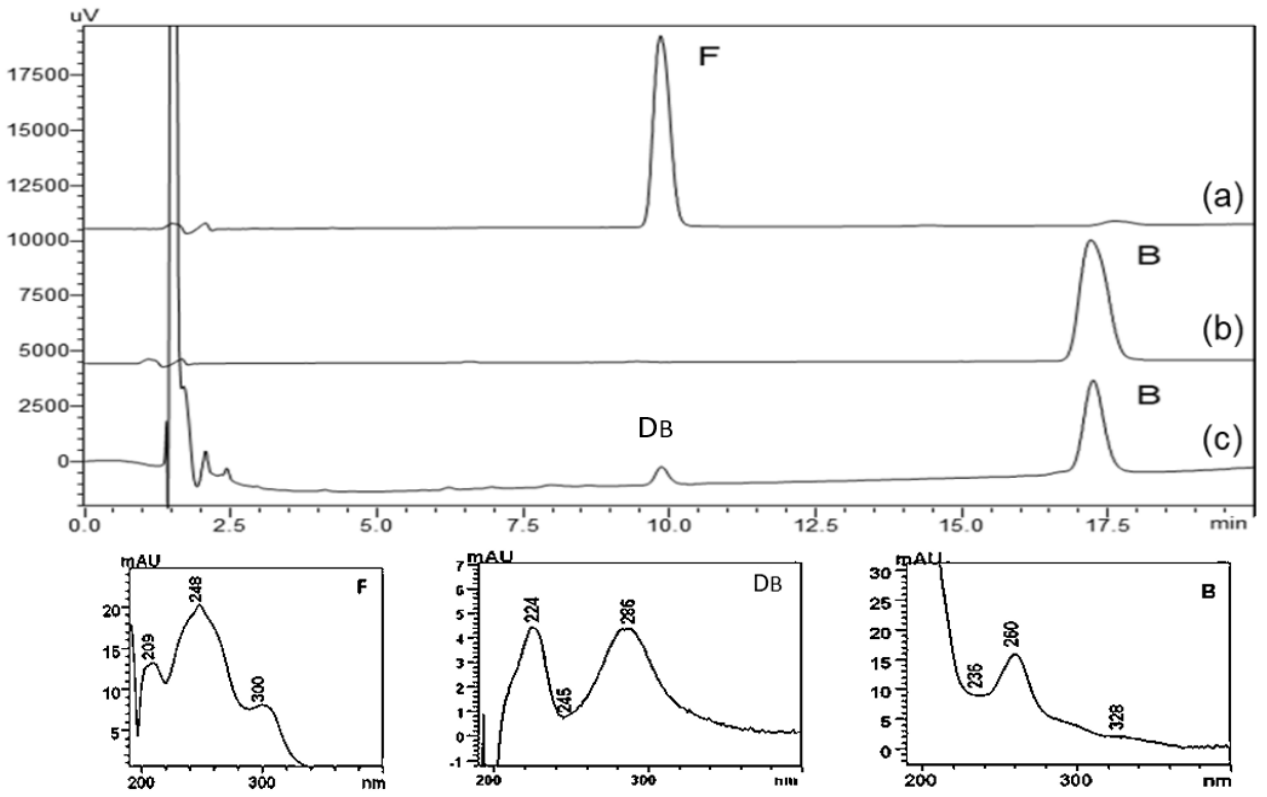

Figure 1. HPLC-DAD chromatograms obtained by the HPLC method reported by Ramos et al. [7]: (a) FORMO standard 5.0 $\mu \mathrm{g} / \mathrm{mL}$; (b) BIO standard $5.0 \mu \mathrm{g} / \mathrm{mL}$; (c) BIO after alkaline degradation $(1 \mathrm{M} \mathrm{NaOH}, 24 \mathrm{~h})$. (F) the peak of FORMO (UV spectrum 248 , $300 \mathrm{~nm}$ ); (B) BIO peak (UV spectrum 260, $328 \mathrm{~nm}$ ); (DB) peak of the alkaline degradation of BIO (UV spectrum $245,286 \mathrm{~nm}$ ). 


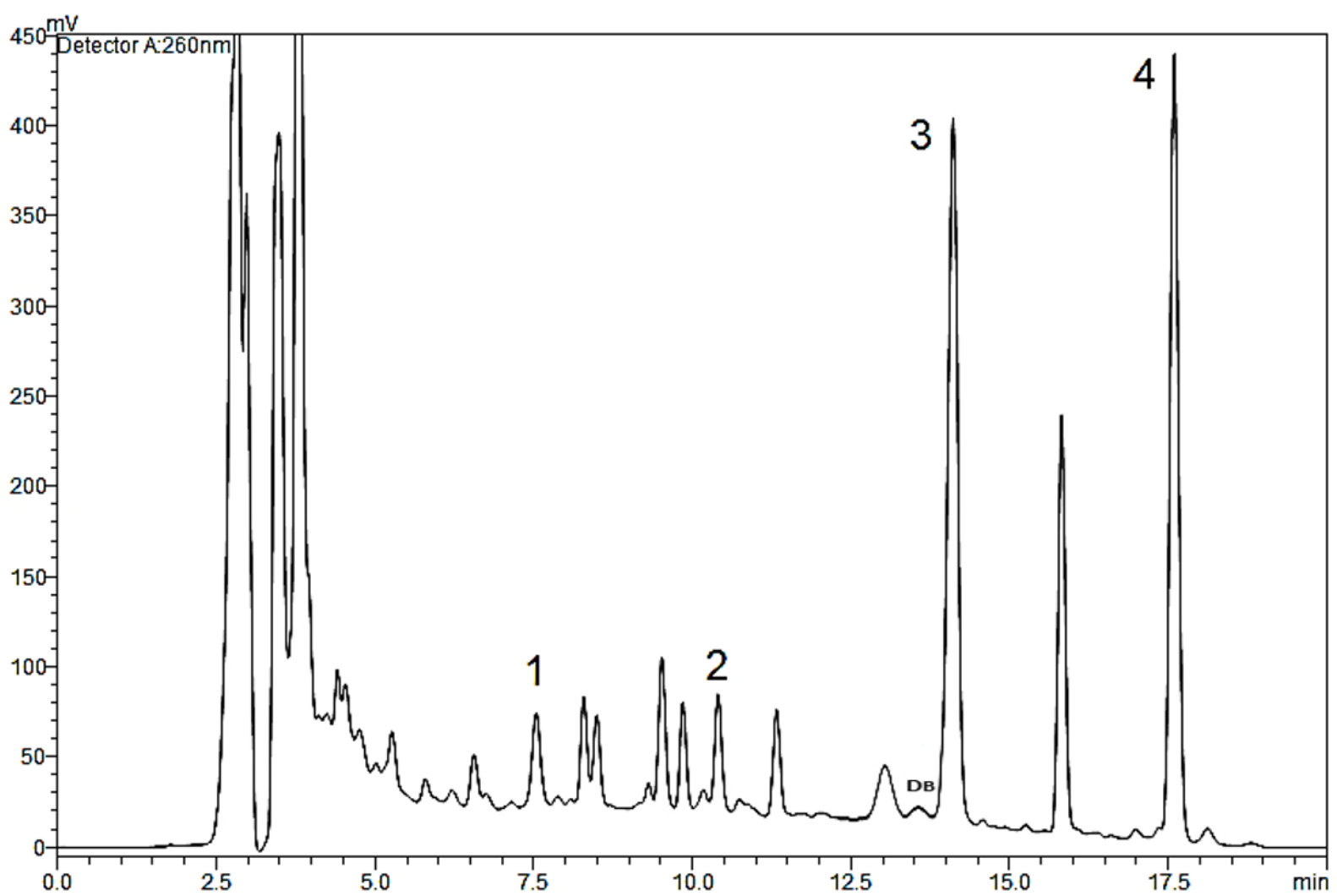

Figure 2. Representative chromatogram of Trifolium pratense extract, added of the $\mathrm{BIO}$ alkaline degradation product, $\mathrm{DB}(\mathrm{NaOH}$ 1M 24h), where: (1) DAID, (2) GEN, (3) FORMO, (4) BIO.

Table 2. HPLC data and molecular ion $[\mathrm{M}+\mathrm{H}]^{+}$acquired by MS analysis (UPLC-QTOF/HDMS) of the major Trifolium pratense isoflavone aglycones.

\begin{tabular}{|c|c|c|c|c|c|c|}
\hline Compound & Chemical Structure & Retention Time & Plates & Tailing Factor & Resolution & {$[\mathbf{M}+1]^{+}$} \\
\hline DAID & & 7.53 & 14039.765 & 1.039 & - & 255 \\
\hline GEN & & 10.39 & 39279.582 & 1.118 & 12.349 & 271 \\
\hline FORMO & & 14.10 & 36476.301 & 0.903 & 2.762 & 269 \\
\hline $\mathrm{BIO}$ & & 17.58 & 77971.943 & 1.094 & 12.721 & 285 \\
\hline
\end{tabular}

HPLC analysis: CLC-ODS (4.6 mm X $25 \mathrm{~cm}$ i.d; 5 $\mu \mathrm{m}$ ) shim-pack column (Shimadzu, Kyoto, Japan) coupled to a C18 pre-column (20 X $3.9 \mathrm{~mm}$ i.d; $10 \mathrm{~mm}$ ); mobile phase A composed of water acidified with $0.1 \%$ of trifluoroacetic acid (TFA) and a phase B composed of acetonitrile; gradient elution was 30\% B (0-2 min), 30-55\% B (2-13 $\mathrm{min}), 55-30 \% \mathrm{~B}(13-19 \mathrm{~min})$; flow rate was a gradient of $1.0 \mathrm{~mL} / \mathrm{min}(0-11.7 \mathrm{~min}), 1.0-0.4$ $\mathrm{mL} / \mathrm{min}(11.7-12.2 \mathrm{~min}), 0.4 \mathrm{~mL} / \mathrm{min}(12.2-13.8 \mathrm{~min}), 0.4-1.0 \mathrm{~mL} / \mathrm{min}(13.8-14.2 \mathrm{~min}), 1.0 \mathrm{~mL} / \mathrm{min}$ (14.2-19 min); injection volume was 10 $\mu \mathrm{L}$; analysis temperature $40^{\circ} \mathrm{C}$ and $\mathrm{UV}$ detector $(260 \mathrm{~nm})$. 


\section{Validation of the HPLC method}

Assessment of the matrix effect

When the analyte is incorporated into a complex matrix compared to a standard or reference solution, the matrix effect on the analyte quantification is a critical parameter that should be evaluated during the validation of an analytical method. This effect can result in suppression or enhancement of the response, which may impair the correct quantification of analytes [15]. The results for the matrix effect (ME\% $<2.24$ ) for each aglycone isoflavone are shown in Table 3. These data indicate that the samples show a low matrix effect (ME\%) for the determination of isoflavones when analyzed according to as recommended by Niessen et al. [19] (low matrix effect for a range between $-20 \%<\mathrm{C} \%<20 \%$ ).

\section{Specificity}

The chromatographic separation (HPLC) of isoflavone aglycones (standards) at $260 \mathrm{~nm}$ showed retention times of 7.49, 10.42, 14.13 and 17.49 min for DAID, GEN, FORMO, and $\mathrm{BIO}$, respectively (Fig. 3A). The $\lambda \max$ spectra corresponding to each peak are shown in figure 3B (HPLCDAD); the mass spectrum with the characteristic molecular ions is shown in Figure 3C, which is consistent with those already reported for this type of molecules $[8,15,18]$.

Table 3. Linearity data of the isoflavone aglycones (standards) and matrix effect in Trifolium pratense extract in the HPLC method.

\begin{tabular}{|c|c|c|c|c|c|c|}
\hline Matrix & Compound & Equation & $\mathbf{R}^{2}$ & $\begin{array}{c}\text { LOD } \\
(\mu \mathrm{g} / \mathrm{mL})\end{array}$ & $\begin{array}{c}\text { LOQ } \\
(\mu \mathrm{g} / \mathrm{mL})\end{array}$ & $\operatorname{ME}(\%)$ \\
\hline \multirow{4}{*}{ Reference substances } & DAID & $y=56170 x+1646,3$ & 0.9999 & 0.053 & 0.159 & - \\
\hline & GEN & $y=65422 x+2531,7$ & 0.9996 & 0.099 & 0.299 & - \\
\hline & FORMO & $y=75562 x-973,55$ & 0.9990 & 0.151 & 0.458 & - \\
\hline & $\mathrm{BIO}$ & $y=65256 x-1638,6$ & 0.9998 & 0.062 & 0.188 & - \\
\hline \multirow{4}{*}{ Trifolium pratense extract } & DAID & $y=55844 x+1666,4$ & 0.9997 & 0.043 & 0.131 & -0.58 \\
\hline & GEN & $y=66412 x-710,24$ & 0.9994 & 0.058 & 0.175 & 1.49 \\
\hline & FORMO & $y=77297 x-2480,9$ & 0.9999 & 0.029 & 0.087 & 2.24 \\
\hline & $\mathrm{BIO}$ & $y=64033 x+354,54$ & 0.9992 & 0.067 & 0.204 & -1.91 \\
\hline
\end{tabular}

$\mathrm{R}^{2}=$ determination coefficient; $\mathrm{LOD}=$ limit of detection; $\mathrm{LOQ}=$ limit of quantitation; $\mathrm{ME}=$ matrix effect.

(a)

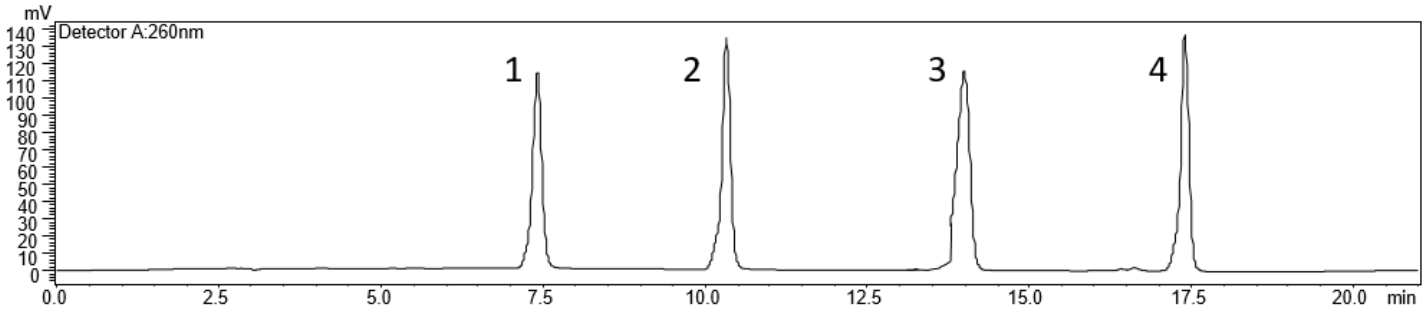

(b)
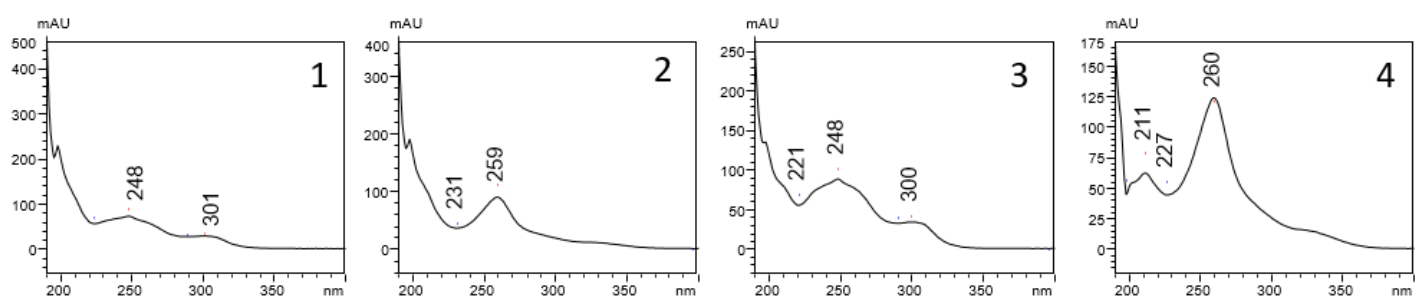

(c)
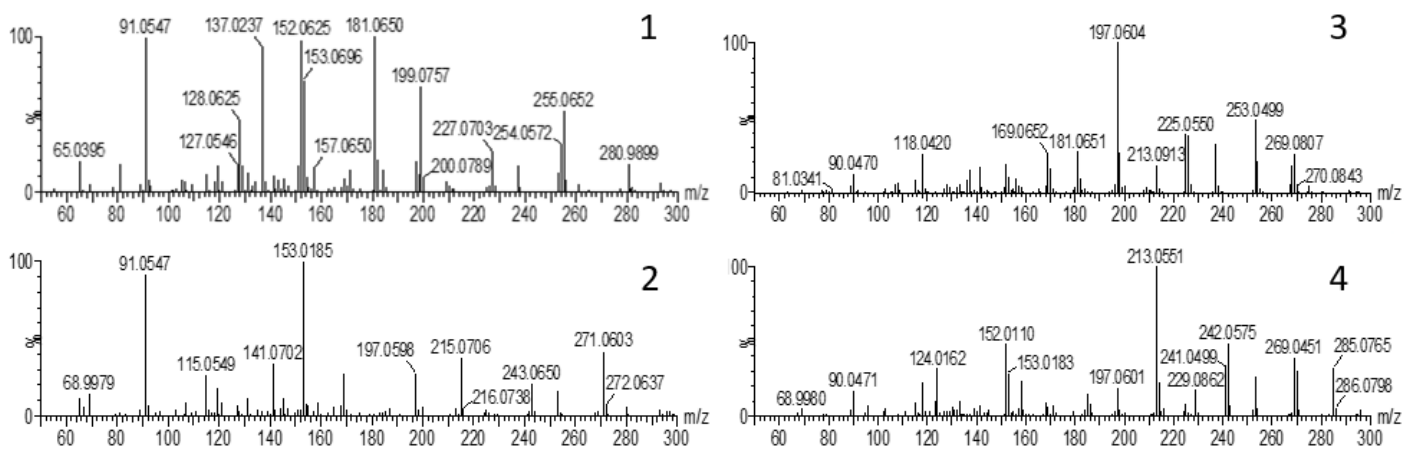

Figure 3. Analysis of isoflavone aglycones (1) DAID, (2) GEN, (3) FORMO, and (4) BIO by (a) HPLC tested method; (b) UV spectra; (c) MS/MS spectra measured in positive mode (ESI+). 
To evaluate the specificity of the HPLC method, samples of Trifolium pratense extract and cyclodextrins were injected in the equipment and the purity of the peaks was checked. Figure 4a shows that all isoflavone peaks exhibit high purity (Fig. 4a) demonstrating the absence on interferences of the other extract matrix constituents as well as the of the tested cyclodextrins (which did not show any signal in the chromatogram). A sample of the cyclodextrin-extract spraydried powder was also evaluated, and the purity of all the analytes was verified, denoting that there was no interference on the four isoflavones quantification.

These data represent critical information for developing of analytical methods as part of a validation protocol and produce valuable information for investigation of degradation products. A well planned and executed forced degradation study provides a representative sample for developing a stability-indicating HPLC method [20].
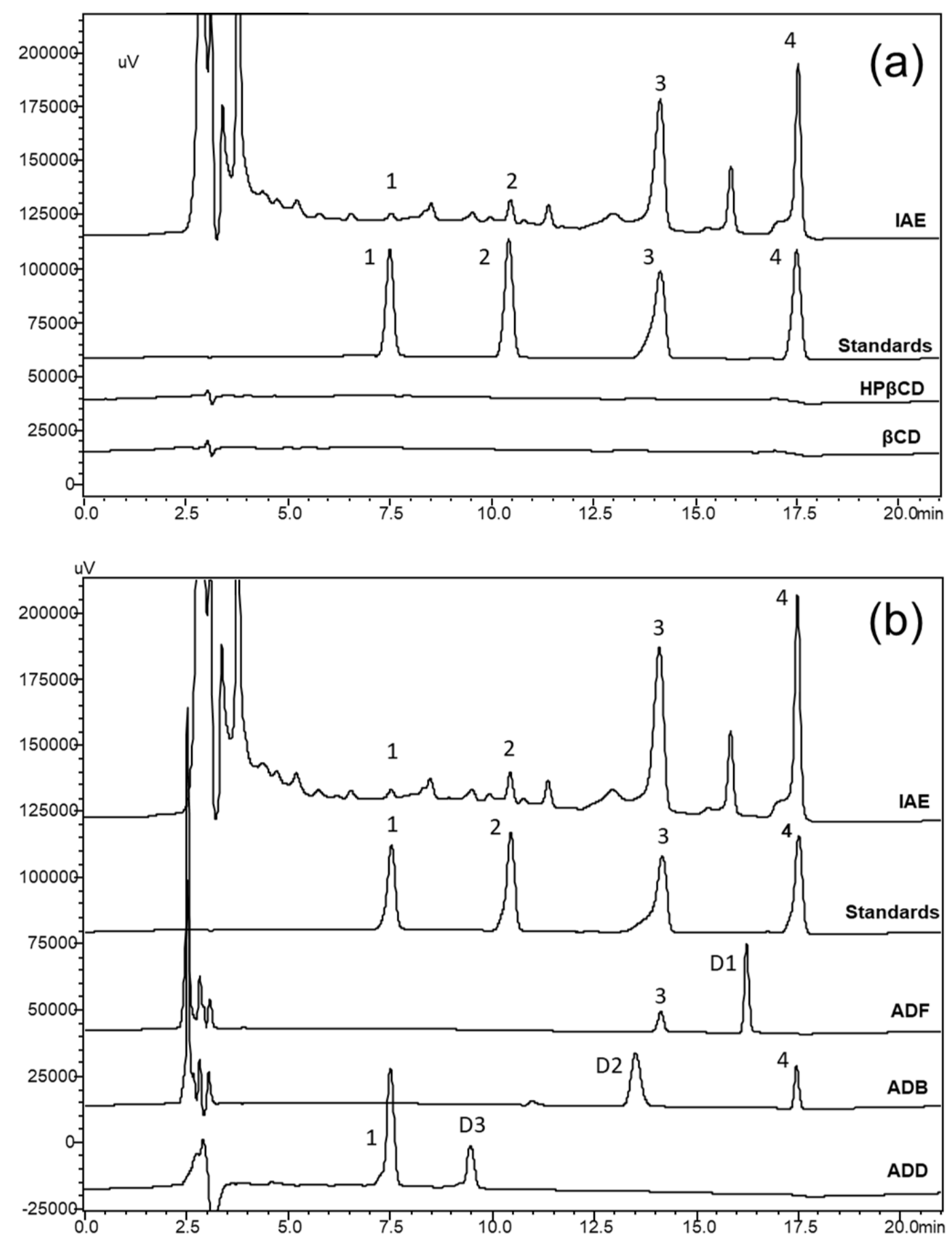

Figure 4. Representative chromatographic profiles obtained in the specificity assay for the: (a) Trifolium pratense and its reference isoflavones and the absence of interference of cyclodextrins in the chromatographic analysis, where, IAE: isoflavone aglycones extract, HP $\beta C D$ : hydroxypropyl-beta cyclodextrin, $\beta C D$ : beta-cyclodextrin; (b) alkaline degradations products from FORMO, BIO, and DAID, were, ADF: peak from alkaline degradation of formononetin, ADB: alkaline degradation of biochanin A, ADD: alkaline degradation of daidzein. Peak 1 = daidzein, Peak 2 = genistein Peak $3=$ formononetin and Peak 4 = biochanin A. 
However, there is no consensus as to how much stress is required to undertake this type of study. Some pharmaceutical scientists suggest degradations between 5 and $20 \%$ as being reasonable for the validation of chromatographic assays [2022]. It is also not necessary for forced degradation to result in a degradation product, but in this study, we performed a screening at different levels of stress (variation of exposure time and variation in the concentration of degrading agent) in an attempt to detect the possible degradation product formation of both at low and slightly higher levels of stress. Even in the most extreme conditions tested, all the aglycone isoflavones (FORMO, BIO, DAID, and GEN) presented a degradation percentage below $20 \%$ (RSD <3.7\%) for thermal, acid and oxidative degradation. On the other hand, in the condition of photolysis, FORMO presented degradation higher than $30 \%$ (RSD $4.5 \%$ ) for both times of exposure (24h, $48 \mathrm{~h}$ ). The forced degradation study revealed that the tested isoflavones gradually degraded in alkaline medium, showing significant variation after $2 \mathrm{~h}$ of stress. After $24 \mathrm{~h}$ of exposure to the alkaline medium, degradation of $98.01 \%$ of FORMO (RSD 3.6\%), $93.19 \%$ of BIO (RSD 3.6\%), $79.97 \%$ of DAID (RSD $3.00 \%$ ), and $66.33 \%$ GEN (RSD 1.9\%).

However, only three new peaks corresponding to the degradation products of FORMO, BIO, and DAID could be observed in the HPLC method (Fig 4B), D1, D2, and D3 which showed retention times of $16.2,13.5$ and $9.4 \mathrm{~min}$, respectively. All degradation products showed good separation from the other peaks of the reference substances and even of the matrix, resulting in a very good resolution (>2.0). The maximum UV spectra of the principal degraded peaks showed $\lambda \max : 245 / 281 \mathrm{~nm}$ for D1, $\lambda \max : 245 / 286 \mathrm{~nm}$ for D2 and $\lambda \max : 281 / 304 \mathrm{~nm}$ for D3.

The stability tests associated with the UPLC-QTOF/HDMS analysis is a new and relevant tool for determining the fragmentation pattern and the chemical structures of the main degradation products. Thus, to increase knowledge of the identity of degradation products of FORMO, BIO, DAID and GEN and to confirm the purity of the aglycone isoflavones, they were analyzed by mass spectrometry (UPLC-QTOF/HDMS), after stress. In this system, the retention times of the peaks were 4.54 (FORMO), 6.21 (BIO), 2.16 (DAID) and $3.37 \mathrm{~min}$ (GEN) for the reference isoflavones and 5.6, 4.5 and $2.9 \mathrm{~min}$ for the degradation products D1, D2, and D3, respectively. We obtained for D1 the precursor $[\mathrm{M}-\mathrm{H}]$ - the ion $\mathrm{m} / \mathrm{z} 257$ and as products the ions 224 and $109 \mathrm{~m} / \mathrm{z}$, which have similarities to those described by Yatsu et al. in 2014 [15] for O-desmethylangolensin.

The proposed molecular formula and the corresponding fragmentation ions are shown in Table 4 for the three alkaline degradation products $(1 \mathrm{M} \mathrm{NaOH})$. However, more studies are necessary to enlighten their chemical structure.

\section{Linearity, precision and accuracy}

The linearity of the response between the peak areas and isoflavone concentrations is presented in Table 3. It was obtained through reference analytical curves determined in three different days, demonstrating good linearity through the analysis of determination coefficients (>0.999) for all compounds within the tested range. The graphic analysis of the residuals and the confidence interval observed in the t-test in the intercepts reveal the absence of constant systematic error. The ANOVA test for regression residue analysis did not show any deviation in the linearity in the analyzed concentration range, and there was no significant variation between the curves on different days ( $p>0.05$ ). The results of LOD and LOQ (table 3 ) show that the method presents good sensitivity for the analyzed standards.

The precision of the method was assessed by determining the repeatability and intermediate precision of the isoflavone aglycone reference in the matrix. Even being a complex matrix, the results (RSD $<5 \%$ ) demonstrate that the method is precise. Intra-day precision data showed a relative standard deviation (RSD) value of $0.47-4.58 \%$ for the lowest concentration level, between $0.33-2.38 \%$ for the average level and between $0.33-2.17 \%$ for the highest level. The inter-day precision data had an RSD value between 1.58$3.79 \%$ for the lowest-level tests, between $0.53-1.95 \%$ for the medium level and between $0.68-1.63 \%$ for the highest level of concentration.

The accuracy of the method was determined using a recovery test. The recovery results were $97.03-104.04 \%$ for the low level, $98.84-100.17 \%$ for the mean level, and 99.14$100.35 \%$, which is considered adequate according to the validation guidelines.

Table 4. Schematic representation of ion mass spectra (MS) of isoflavone degradation products in alkaline media (1M NaOH) and proposed molecular formula.

\begin{tabular}{clcccccccc}
\hline Degradation product & Precursor & {$[\mathbf{M}-\mathbf{H}]-$} & {$[\mathbf{M}-\mathrm{H}]+$} & \multicolumn{2}{c}{ Neutral Monoisotopic mass } & $\Delta(\mathbf{p p m}) *$ & Molecular formula \\
& & & & Determined & Theoretical & \\
\hline D1 & FORMO & 257.0819 & 259.0971 & 258.0895 & 258.0892 & 1.16 & C15H14O4 \\
D2 & BIO & 273.0764 & 275.0923 & 274.0844 & 274.0841 & 0.91 & C15H14O5 \\
D3 & DAID & 243.0659 & 245.0806 & 244.0733 & 244.0736 & -1.43 & C14H12O4 \\
\hline
\end{tabular}

\footnotetext{
*mass error ppm $\quad{ }^{\mathrm{a}}$ obtained from isotopic pattern
} 
Robustness Analysis

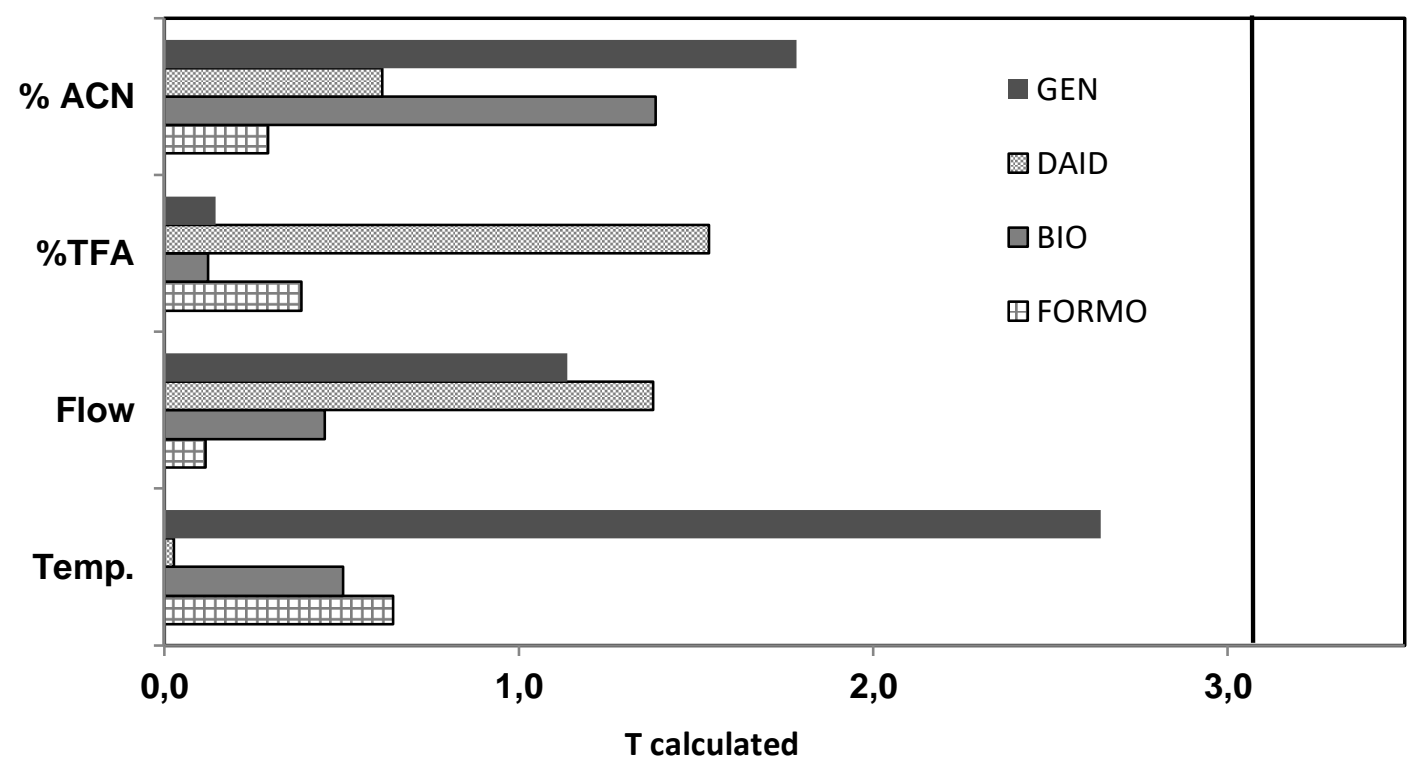

Figure 5. Bar charts representing the t-calculated for quantitative determination (assay) of the investigated factors $(1,2,3$, and 4$)$ in Plackett-Burman experimental design and their t-critical, represented by the vertical line, for each isoflavone in the matrix (Trifolium pratense extract).

\section{Robustness}

Robustness is critical to assess if the analytical method developed can remain unaffected to small, deliberate variations in the method parameters and consequently ensures that the method is reliable during routine use of analyses $[12,23]$. The Plackett-Burman screening design was used as statistical tool for robustness assessment since several factors can be analyzed simultaneously with a reduced number of experiments [24].

From the experimental design, the percentage of FORMO, BIO, DAID, and GEN in the samples was calculated in relation to the reference solutions for each experiment. The results show that there was no difference of significance for all the analyses (Fig. 5), where the calculated $t$ values were lower than the critical t $(\alpha=0.05)$. Therefore, the deliberate variations in the experimental conditions did not interfere with the percentage of aglycone isoflavones, demonstrating the robustness of the proposed analytical method.

\section{System suitability}

Routine analysis of the reference substances was performed under the developed experimental conditions and the following values and their variability (RSD \%) for each compound were evaluated: (I) DAID analysis: 7.49 (0.08) min for migration time, 6943.63 (1.21) for plates, and 0.998 (1.15) for tailing factor; (II) GEN analysis: 10.42 (0.03) $\mathrm{min}$ for migration time, plates 12104.80 (1.61), and tailing factor 0.962 (0.95); (III) FORMO analysis: 14.13 (0.04) min for migration time, 12516.68 (4.23) for plates, and $0.730(0.42)$ for tailing factor; (IV) BIO analysis: $17.49(0.05) \mathrm{min}$ for migration time, 33186.34 (1.72) for plates, and 0.948 (0.57) for the tailing factor. The resolution between DAID and GEN peaks was 7.92 (0.64), GEN and FORMO peaks was 8.39 (1.49) and 7.55 (1.10) between FORMO and BIO. The parameters indicate that the system is suitable for the analysis.

\section{Stability of the aglycones isoflavones in the matrix}

In the study evaluating the stability of the isoflavones in the matrix, they demonstrated no need to change their concentration in the matrix after $48 \mathrm{~h}$ storage at room temperature (RSD $<5.0 \%$ ).

The variation in the values of the isoflavones concentration in the matrix, after this period, were found to be between 99.25 and $103.91 \%$ (RSD 1.94\%) for FORMO, 99.68 to $103.16 \%$ (RSD $1.63 \%$ ) for BIO, between 98.31 to $103.46 \%$ (RSD $2.32 \%$ ) for DAID and between 98.24 to $104.77 \%$ (RSD $2.68 \%$ ) for GEN.

\section{Method applications}

For verifying the applicability of the method, samples of products derived from Trifolium pratense were tested. The content of isoflavone aglycones present in Trifolium pratense extract (TPE), an isoflavone-enriched fraction (IEF), and an extract of Trifolium pratense associated to $\beta C D$ (TPE: $\beta C D)$ was determined. The results (Table 5) indicate the accuracy of the method in all samples, where the RSD was less than $3.26 \%$ in the test for TPE, $3.21 \%$ for IEF and 4.24 for TPE: $\beta C D$. 
Table 5. Applicability of the HPLC validated method: determination of the isoflavone aglycones in different matrices.

\begin{tabular}{llll}
\hline & \multicolumn{3}{c}{$\begin{array}{c}\text { Concentration in } \mu \mathrm{g} / \mathrm{mL} \text { (RSD \%) } \\
\text { mean of six replicates }\end{array}$} \\
\cline { 2 - 4 } & TPE & IEF & TPE: $\beta C D$ \\
\hline FORMO & $69.19(3.26)$ & $147.94(3.21)$ & $59.12(4.24)$ \\
BIO & $79.63(0.64)$ & $207.57(1.52)$ & $44.03(2.78)$ \\
DAID & $2.75(2.87)$ & $1.39(2.77)$ & $0.60(1.35)$ \\
GEN & $7.04(2.54)$ & $9.31(3.88)$ & $1.86(2.90)$ \\
$\begin{array}{l}\text { Total } \\
\text { isoflavones }\end{array}$ & $\mathbf{1 5 8 . 6 1 ( 1 . 3 4 )}$ & $\mathbf{3 7 2 . 4 6}(\mathbf{2 . 5 4})$ & $\mathbf{1 0 5 . 6 1 ( 0 . 8 8 )}$ \\
\hline
\end{tabular}

TPE: Trifolium pratense extract; IEF: (isoflavone-enriched fraction) and TPE: $\beta C D$ : Trifolium pratense extract complexed with $\beta$-cyclodextrin.

\section{Conclusions}

This is the first report on a validated stability-indicating HPLC method capable of quantifying the four isoflavones from Trifolium pratense, formononetin, biochanin A, genistein, and daidzein. The method is fast and selective against the other extract constituents, and the degradation products generated in alkaline forced conditions. The method showed robustness, low matrix effect, linearity, precision, and accuracy. The UPLC-QTOF/HDMS fragmentation pattern and a molecular formula of three degradation products from forced degradation were determined. In summary, the proposed stability-indicating HPLC validated method allows the simultaneous quantification of the four isoflavones of Trifolium pratense in a fast and reliable way, which is useful for application in food or pharmaceutical fields.

\section{Acknowledgments}

The authors are gratefully acknowledged to Conselho Nacional de Desenvolvimento científico e Tecnológico (CNPq0, Fundação de Amparo à Pesquisa do Estado do Rio Grande do Sul (FAPERGS), and Coordenação de Aperfeiçoamento de Pessoal de Nível Superior - Brasil (CAPES - Finance Code 001) for the financial grants. S.M. would like to thank CAPES for the doctoral grant. V.L.B. is recipient of $\mathrm{CNPq}$ research fellowship.

\section{Conflict of interest}

The authors declare no conflicts of interest.

\section{References}

1. Silva AG. Plantas contendo isoflavonas no tratamento da síndrome da menopausa e nos distúrbios do climatério. Nat. Line 2007;5:25-9.

2. Barnes J, Anderson L, Phillipson J. Fitoterápicos. Porto Alefre: 2012.

3. Kaurinovic B, Popovic M, Vlaisavljevic S, Schwartsova $\mathrm{H}$, Vojinovic-Miloradov M. Antioxidant profile of Trifolium pratense L. Molecules 2012;17:11156-72.

4. Ramos GP, Apel MA, Morais CB de, Ceolato PC, Schapoval EES, Dall'Agnol M, et al. In vivo and in vitro anti-inflammatory activity of red clover Trifolium pratense dry extract. Rev. Bras. Farmacogn. 2012;22:176-80.

5. Kolodziejczyk-Czepas J. Trifolium species-derived substances and extracts-Biological activity and prospects for medicinal applications. J. Ethnopharmacol. 2012;143:14-23.

6. Chen Q, Li P, Li B, Xiulou, Zhu J, Chen F. Simultaneous determination of formononetin, biochanin A, daidzein, and genistein in Trifolium pratense (red clover) by HPLC. LC GC Eur. 2010;23.

7. Ramos GP, Dias PMB, Morais CB, Fröehlich PE, Dall'Agnol M, Zuanazzi J a. S. LC determination of four isoflavone aglycones in red clover (Trifolium pratense L.). Chromatographia 2008;67:125-9.

8. Wu Q, Wang M, Simon JE. Determination of isoflavones in red clover and related species by high-performance liquid chromatography combined with ultraviolet and mass spectrometric detection. J. Chromatogr. A 2003;1016:195-209.

9. Oleszek W, Stochmal A, Janda B. Concentration of isoflavones and other phenolics in the aerial parts of Trifolium species. J. Agric. Food Chem. 2007;55:8095100 .

10. Lin LZ, He XG, Lindenmaier M, Yang J, Cleary M, Qiu SX, et al. LC-ESI-MS study of the flavonoid glycoside malonates of red clover (Trifolium pratense). J. Agric. Food Chem. 2000;48:354-65.

11. Saviranta NM, Anttonen MJ, von Wright A, Karjalainen RO. Red clover (Trifolium pratense L.) isoflavones: determination of concentrations by plant stage, flower colour, plant part and cultivar. J. Sci. Food Agric. 2008;88:125-32.

12. ICH. Validation of a analytical Procedures: text and methodology Q2(R1). In: Guidance. 2005. page 17. 
13. FDA F and DA. Guidance for industry: bioanalytical method validation, 2013.

14. Watanabe E, Kobara Y, Baba K, Eun H. Aqueous acetonitrile extraction for pesticide residue analysis in agricultural products with HPLC-DAD. Food Chem. 2014;154:7-12.

15. Yatsu FKJ, Pedrazza GPR, Argenta DF, Barreto F, Nemitz MC, Teixeira HF, et al. A new simplified and stability indicating liquid chromatography method for routine analysis of isoflavones aglycones in different complex matrices. Food Anal. Methods 2014;7:1881-90.

16. Vander Heyden Y, Nijhuis A, Smeyers-Verbeke J, Vandeginste BG, Massart DL. Guidance for robustness/ruggedness tests in method validation. $J$ Pharm Biomed Anal. 2001;24(5-6):723-753. doi:10.1016/s0731-7085(00)00529-x

17. de Rijke E, Zafra-Gómez A, Ariese F, Brinkman UAT, Gooijer C. Determination of isoflavone glucoside malonates in Trifolium pratense L. (red clover) extracts: quantification and stability studies. J. Chromatogr. A 2001;932:55-64.

18. Nemitz MC, Yatsu FKJ, Bidone J, Koester LS, Bassani VL, Garcia C V., et al. A versatile, stability-indicating and high-throughput ultra-fast liquid chromatography method for the determination of isoflavone aglycones in soybeans, topical formulations, and permeation assays. Talanta 2015; 134:183-93.
19. Niessen WMA, Manini P, Andreoli R. Matrix effects in quantitative pesticide analysis using liquid chromatography-mass spectrometry. Mass Spectrom. Rev. 2006;25:881-99.

20. Shah B, Jain S, Prajapati K, Mansuri N. Stability indicating HPLC method development: A review. Int. J. Pharm. Sci. Res. 2012;3:2978-88.

21. Blessy M, Patel RD, Prajapati PN, Agrawal YK. Development of forced degradation and stability indicating studies of drugs-A review. J. Pharm. Anal. 2014;4:159-65.

22. Carr GP, Wahlich JC. A practical approach to method validation in pharmaceutical analysis. J. Pharm. Biomed. Anal. 1990;8:613-8.

23. Anvisa. RDC N. 166, de 25 de Julho de 2017, Dispõe sobre a validação de métodos analíticos e dá outras providências. Minist. da Saúde 2017;2017:1-21.

24.Zeaiter M, Roger JM, Bellon-Maurel V, Rutledge DN. Robustness of models developed by multivariate calibration. Part I: The assessment of robustness. TrAC Trends Anal. Chem.2004;23:157-70. 